\title{
Scalable Telehealth Services to Combat Novel Coronavirus (COVID-19) Pandemic
}

\author{
Shah Muhammad Azmat Ullah ${ }^{1}$ Md. Milon Islam² ${ }^{2} \cdot$ Saifuddin Mahmud $^{3} \cdot$ Sheikh Nooruddin $^{2}$. \\ S. M. Taslim Uddin Raju ${ }^{2} \cdot$ Md. Rezwanul Haque $^{2}$
}

Received: 28 October 2020 / Accepted: 18 November 2020 / Published online: 6 January 2021

(c) Springer Nature Singapore Pte Ltd 2021

\begin{abstract}
An ongoing pandemic, the novel coronavirus disease 2019 (COVID-19) is threatening the nations of the world regardless of health infrastructure conditions. In the age of digital electronic information and telecommunication technology, scalable telehealth services are gaining immense importance by helping to maintain social distances while providing necessary healthcare services. This paper aims to review the various types of scalable telehealth services used to support patients infected by COVID-19 and other diseases during this pandemic. Recently published research papers collected from various sources such as Google Scholar, ResearchGate, PubMed, Scopus, and IEEE Xplore databases using the terms "Telehealth", "Coronavirus", "Scalable" and "COVID-19" are reviewed. The input data and relevant reports for the analysis and assessment of the various aspects of telehealth technology in the COVID-19 pandemic are taken from official websites. We described the available telehealth systems based on their communication media such as mobile networks, social media, and software based models throughout the review. A comparative analysis among the reviewed systems along with necessary challenges and possible future directions are also drawn for the proper selection of affordable technologies. The usage of scalable telehealth systems improves the quality of the healthcare system and also reduces the infection rate while keeping both patients and doctors safe during the pandemic.
\end{abstract}

Keywords COVID-19 $\cdot$ Telehealth $\cdot$ Telemedicine $\cdot$ Mobile network $\cdot$ Social media $\cdot$ Software

This article is part of the topical collection "Advances in

Computational Approaches for Artificial Intelligence, Image

Processing, IoT and Cloud Applications" guest edited by Bhanu Prakash K N and M. Shivakumar.

Md. Milon Islam

milonislam@cse.kuet.ac.bd

Shah Muhammad Azmat Ullah

azmat.ece.kuet@gmail.com

Saifuddin Mahmud

smahmud2@kent.edu

Sheikh Nooruddin

nooruddinimad@gmail.com

S. M. Taslim Uddin Raju

taslimuddinraju7864@gmail.com

Md. Rezwanul Haque

rezwanh001@gmail.com

\section{Introduction}

COVID-19 is a highly contagious disease caused by a novel coronavirus which was discovered at the end of December 2019. According to the World Health Organization (WHO), it has become a pandemic in recent times. This pandemic has affected the entire world and forced us to reconsider the

1 Department of Electronics and Communication Engineering, Khulna University of Engineering \& Technology, Khulna 9203, Bangladesh

2 Department of Computer Science and Engineering, Khulna University of Engineering \& Technology, Khulna 9203, Bangladesh

3 Advanced Telerobotics Research Lab, Department of Computer Science, Kent State University, Kent, Ohio, USA 
available healthcare facilities, treatment systems and technologies [1-3].

Social gap or social distancing is the major way of reducing the transmission of the virus. Social distance is made possible by decreasing the person to person contact $[4,5]$. To reduce mass transmissions, travel restrictions have been established and enforced around the world, and most of the cities have been quarantined. In this situation, it is difficult to take treatment from specialists at the clinic or hospital in person $[6,7]$. In the age of modern technology of mobile communication [8-10] and virtual reality [11, 12], telehealth is the ideal system for such kind of communicable disease [13]. The evolution of telecommunication technologies plays a key role in the ability of telehealth services to exchange valid information for diagnosis and management of diseases and injuries $[14,15]$. There are various types of telehealth systems based on the working principle and method. In a typical telehealth system, patients and doctors are connected by a media which is used to transfer patient medical data to doctor and necessary prescriptions are delivered to the patient. Medical data from patients is acquired by using different types of Body Sensor Network (BSN). Scalability refers to the property of a system to accommodate growing amounts of work by adding resources to the system. Scalable telehealth systems can accommodate the rapidly growing number of coronavirus patients by introducing more resources such as doctors, specialists, artificial assistants, etc. A typical scalable telehealth service with proper functionality is depicted in Fig. 1.
This paper aims to review the available scalable telehealth systems that have been evolved to ensure remote healthcare support for COVID-19 and other disease infected patients. The available frameworks are described here as mobile networks, social media, and software-based systems. All of the systems are demonstrated in terms of working principle, technology, hardware equipment like sensors used, types, and methodology of service provided. A comparative analysis among the available systems with challenges and possible future trends are also presented in the review.

The rest of the paper is organized as follows. The following section describes the existing systems for telehealth services for the patients during the COVID-19 outbreak. The next section describes a relative analysis among the available technologies with the necessary challenges and possible future directions. The subsequent section concludes the paper.

\section{Telehealth Systems}

Telehealth systems are used to deliver health-related services and medicare in such a pandemic where reducing the infection caused by close contact is an important fact $[16,17]$. Similarly, in the COVID-19 pandemic, telehealth systems reduce the infection rate while the patients are allowed to continue their diagnostic-therapeutic process $[18,19]$. The most used telehealth systems which are utilized for COVID19 as well as other diseases can be categorized into three
Fig. 1 Typical functionality of the telehealth system

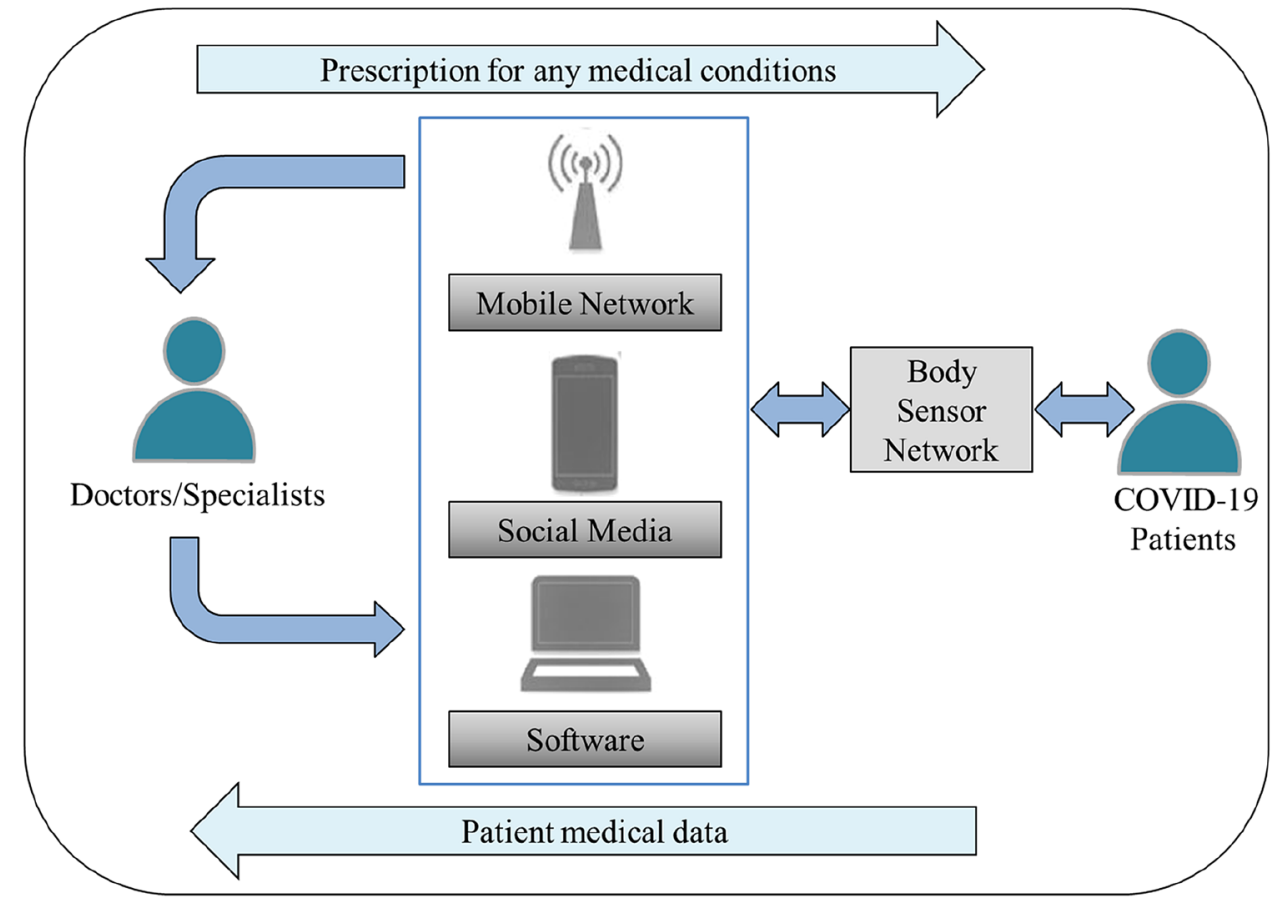


types based on their communication media. Figure 2 shows the possible categorization for easy understanding.

\section{(A) Mobile Network Based Systems}

There are various telehealth systems available using the mobile network which are frequently used in the COVID-19 pandemic [20, 21]. In a mobile network based scalable telehealth system, the data from patients is acquired and transmitted through a wireless communication network as well as the Global System for Mobile Communication (GSM) networks [22, 23].

García and Torres [24] proposed a telehealth system using a commercial GSM network. The developed system measured temperature, heart rate, respiratory rate, and blood pressure using the Body Sensor Network, which are the most important parameters for monitoring the condition of COVID-19 patients. It consists of three subsystems (1) Input subsystem to collect medical information, (2) Local subsystem to transmit data using GSM cellular network, and (3) Remote subsystem to receive and show on a website. A compact and user-friendly telehealth system was developed by Suganthi et al. [25] using GSM/General Packet Radio Service (GPRS), global positioning system (GPS), and peerto-peer (P2P) technology. The proposed system consists of a temperature sensor, Blood Pressure (BP) measurement sensor, pulse counter clip, and non-invasive glucose monitoring sensor. Using the GPS module, the location of patients and the necessary data are saved in the web server and delivered through a short message service (SMS) to the pre-configured doctor's phone. For continuous monitoring, if any abnormality is found, then an emergency flag is raised to the doctors

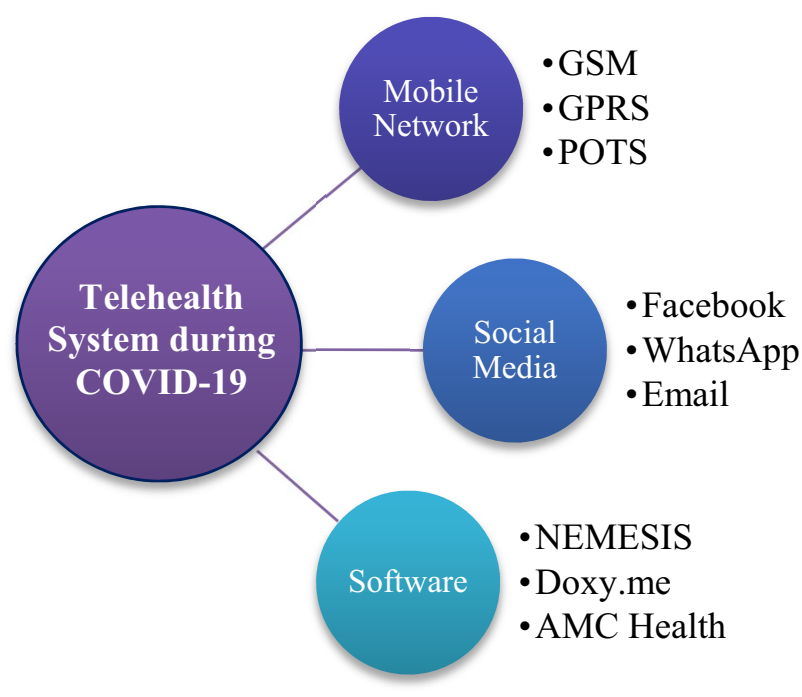

Fig. 2 Categorization of telehealth systems used in the COVID-19 outbreak automatically. Besides, in emergency cases, an automatic call is generated to the doctor and the doctor can hear any voice or messages from the site of the patient. During the COVID-19 pandemic situation, this type of telehealth system can help in early and distance treatment for COVID-19 and other patients. Using similar technologies, TeleHealth Emergency (THE) system is implemented in [26]. In this system, a tele-bio wristwatch is used which consists of some sensors like BP, pulse rate, temperature, and GSM/GPRS and GPS module. The developed watch works like a telephone for the GSM module and can locate the patient's position using GPS. A speaker and call button in this watch help to make emergency call and a red alert button notify the patient for abnormal BP, heart rate, or temperature. In primary treatment, THE can play an important role.

A 3G cellular network based telehealth system is presented in [27]. The described system has services to provide Electrocardiography (ECG), blood sugar, oxygen saturation ( $\mathrm{SpO} 2)$, and patient health record database to the doctor, pharmacist, technician, social worker, etc. using a $3 \mathrm{G}$ network system. In another research, a novel architecture is presented in [28] using the GSM module. The proposed system consists of some sensor transducers, Analog to Digital (A/D) converter, an embedded processor, a transceiver in a sensor node, and a GSM shield interface, Arduino, and an encoder as a $5 \mathrm{G}$ enhancer. The collected data is routed via GSM voice and control channels to the doctor. Due to the COVID-19 outbreak and lockdown situation, the treatment of other diseases is also being delayed. The developed system can provide proper facilities to the patient having a serious heart problem. A GSM mobile communication network based system is proposed in [29] which is used to provide an emergency diagnosis to COVID-19 patients. The system allows transmission of various bio-signal like 3-12 lead ECG signal, SpO2, noninvasive blood pressure amplifier (NIBP), invasive blood pressure (IBP), and temperature through the GSM network. The still images of patients are also transmitted using the proposed system. While GSM is not available, the transmission is performed through satellite links and plain old telephony system (POTS). Using this device, the doctor can telemetrically move to the patient and treat them properly. The available telehealth systems based on mobile networks are summarized in Table 1.

\section{(B) Social Media Based Systems}

The social media platform is being used widely in the outbreak situation of COVID-19 due to the high availability and user-friendliness of the platforms [30,31]. In this type of telehealth system, doctors and other specialized radiologists are connected to such a social platform [32, 33]. They can monitor the patient's regular condition and prescribe them via message or video call. 
Table 1 Available telehealth services based on mobile networks

\begin{tabular}{|c|c|c|c|c|}
\hline Authors & Received data format & Transferring media & $\begin{array}{l}\text { Major technology/sensor } \\
\text { used }\end{array}$ & Provided services \\
\hline García and Torres [24] & SMS & GSM network & Body sensor network & $\begin{array}{l}\text { Temperature, heart rate, } \\
\text { respiratory rate, and blood } \\
\text { pressure }\end{array}$ \\
\hline Suganthi et al. [25] & SMS & GSM, GPRS, GPS, P2P & $\begin{array}{l}\text { Temperature sensor, blood } \\
\text { pressure measurement } \\
\text { sensor, pulse counter clip, } \\
\text { and non-invasive glucose } \\
\text { monitoring sensor, GPS } \\
\text { module }\end{array}$ & $\begin{array}{l}\text { Temperature, blood pressure, } \\
\text { pulse rate, and non-invasive } \\
\text { glucose monitoring }\end{array}$ \\
\hline Ade et al. [26] & Voice call & GSM, GPS & $\begin{array}{l}\text { BP sensor, pulse rate sensor, } \\
\text { temperature sensor, and } \\
\text { GSM/GPRS and GPS } \\
\text { module }\end{array}$ & $\mathrm{BP}$, pulse rate, temperature \\
\hline Sorwar and Ali [27] & Image, call & 3G cellular network & $\begin{array}{l}\text { ECG, blood sugar, } \mathrm{SpO} 2 \\
\text { sensor }\end{array}$ & ECG, blood sugar, $\mathrm{SpO} 2$ \\
\hline Mishra and Agrawal [28] & Voice call/image & $\begin{array}{l}\text { GSM voice and control } \\
\text { channels }\end{array}$ & $\begin{array}{l}\text { A/D converter, embedded } \\
\text { processor, a transceiver in } \\
\text { a sensor node, GSM shield } \\
\text { interface, Arduino, and an } \\
\text { encoder }\end{array}$ & Serious heart problem \\
\hline Kyriacou et al. [29] & Image, call & GSM or POTS & $\begin{array}{l}\mathrm{ECG}, \mathrm{SpO} 2, \mathrm{NIBP}, \mathrm{IBP} \text {, and } \\
\text { temperature sensor }\end{array}$ & $\begin{array}{l}\text { ECG signal, SpO2, NIBP, IBP, } \\
\text { and temperature }\end{array}$ \\
\hline
\end{tabular}

Recently in Iran, a social media based system [34] is proposed based on WhatsApp and email platform. The image or video of patients is delivered in JPG or MP4 version to the volunteer of the developed system. The volunteers post these images to a private group of radiologists and specialists where the confidentiality of data is maintained properly. Only some registered volunteers and coordinator radiologists accessed this private group for generating reports for certain cases. A preliminary report is delivered to patients in image format through email or WhatsApp message having necessary data and instructions. The system is developed only for chest image now. The developed system can deliver faster service of teleradiology and can eliminate the need for overpopulated hospitals. Besides, a video conferencing and email based telehealth system is described in [35]. The developed system is proposed for adult patients. This study provides consultation or advice to health providers through email or video conferencing. The use of public transportation is eliminated by using this system.

In addition, a new web based system [36] is proposed using Facebook like plug-in architecture. The Facebook app helped users to develop virtual support groups. Friends are searched using different parameters, including name, hobbies, and conditions of health. Many apps will not disclose the real health problems and users should not use their actual name due to privacy reasons. Users can send requests to friends and accept or reject requests from other users. Similarly, Care Deliver Frame (CDF) [37] is one kind of telehealth system based on social networking. The developed system is designed for older adults having a communication platform with their family members. Data from various sensors or devices such as blood pressure/glucose meters and weight scales are transmitted to mobile using Bluetooth. The collected data are posted on the patient's Facebook timeline via message, photo, or video module. Children's family members or pre-allocated professional caregivers access this data via mobile or computer from the Facebook database under appropriate Facebook privacy settings. 'Comm \& Care' app is used to retrieve vital signs measurement data from the patient's timeline by professional caregivers. HC3i.cn [38] is another social media platform for telehealth extensively used in China. The first internet-based platform focuses on health informatics, internet-based medicine, and mHealth in China. The patients can receive consultation from physicians at home. The social media based telehealth systems that are frequently used are shown in Table 2.

\section{(C) Software Based Systems}

In the era of the Internet of Things (IoT), a software based telehealth system is not a new concept. In certain instances, video cameras or other acquisition panels are required as additional equipment in telehealth software [39, 40]. During the COVID-19 pandemic, such type of telehealth system can play a significant role in faster treatment [41]. 
Table 2 Available telehealth services based on social media

\begin{tabular}{lllll}
\hline Authors & Received data format & Transferring media & $\begin{array}{l}\text { Major technology/sensor } \\
\text { used }\end{array}$ & Provided services \\
\hline $\begin{array}{llll}\text { Davarpanah et al. [34] } \\
\text { Nicol et al. [35] }\end{array}$ & $\begin{array}{l}\text { Image } \\
\text { Video }\end{array}$ & $\begin{array}{c}\text { WhatsApp and email } \\
\text { Email or Video Conferenc- } \\
\text { ing }\end{array}$ & N/A & N/A \\
Dhillon et al. [36] & Facebook post & Facebook & N/A & $\begin{array}{c}\text { Chest problem } \\
\text { Any types of problems of } \\
\text { adult patients }\end{array}$ \\
Huang and Hsu [37] & Message, video or image & Facebook & BP, glucose, weight measure & $\begin{array}{c}\text { Older adult patient } \\
\text { adult patients }\end{array}$ \\
Leung et al. [38] & $\begin{array}{l}\text { Message, call, video or } \\
\text { image }\end{array}$ & HC3i.cn & N/A & Any types of patients \\
& & &
\end{tabular}

N/A: not appropriately defined

Several telehealth services are available through software nowadays. Magdalena et al. [42] presented a new telemedicine application named NEMESIS using Java programming language. The developed system can acquire, visualize, and revise the ECG signal and transmit it among several medical stations. It has video conference and electronic mail module to communicate with specialists. The main feature of this system is that it has knowledge database access where all the records are saved and encrypted. Recently, VITASENIOR-MT [43] is introduced as another platform of telehealth service. The proposed system has three parts including a wireless sensor network (WSN), VITABOX, and cloud. VITABOX which is one kind of medical equipment collects data from the patient's body using Bluetooth Low Energy (BLE) biometric sensors. Blood pressure, heart rate, weight, oximetry, and blood glucose are measured by these sensors and sent it to the cloud. Only associated doctors can access these data from the cloud using any web browser. A new telehealth system is designed and implemented for prenatal management in [44]. The developed system consists of a server, prenatal data assistant, and a workstation for an electronic health record. At first, the patient is required to complete registration with email and password through payment transactions. Then, the software collects data such as present and past medical history, family member history, menstrual history, obstetric history, gynecological history, and immunization history from the patient. The main part of this software is the routine checkup part which is important for good patient monitoring. Doctors or specialists check patient data from the website and make teleconsultation and prescription to the patients. In the ongoing COVID-19 pandemic situation, this type of telehealth system can play a vital role in the treatment of obstetrics patients.

Matsumoto et al. [45] described another platform of telehealth based on the feedback control system. The proposed system can share information among clients, clinicians, and medical professionals. The key specification of this system is obtaining and transferring the information of the client, finding a problem, and confirming client safety, informing urgency to co-medical and clinician, and providing health care advice. An object-oriented method named Unified Modeling Language (UML) is used to develop this software. Similarly, a modern telehealth scheme is introduced in [46] based on neurofuzzy technology. It consists of a tele-practitioner terminal, a patient terminal, and a web-based central station. In both the tele-practitioner terminal and the patient terminal, Embedded Expert System (EMES) is used to serve the embedded intelligent agent for telecommunication and information management. Besides, in the central station, a database and EMES modules are used to store the patient's personal information and medical data. XML is used between database and terminal for data transmission. The most popular, simple, and safe telehealth software in recent times is "Doxy.me" [47]. It has a free trial version as well as a paid version. The key features of this site are video conferencing, one-to-one messaging, group calling, screen sharing, and file-sharing options between clients and specialists. This web-based telehealth platform is integrated with Electronic Health Records (EHR) and Practice Management (PM) software. In another research, advanced telehealth software "AMC Health" [48] is developed for health monitoring via the Food and Drug Administration (FDA) approved medical instruments. It involves monitoring and Bluetooth communication, which can be provided either by the patient or caregiver to allow remote devices to work. In the COVID19 situation, AMC Health provides probable diagnosis using their kit that includes Bluetooth at home peripheral devices and smart devices tablets for patients requiring e-visit. The popular telehealth softwares that are widely used is summarised in Table 3.

\section{Discussions}

In this review, a brief discussion on the scalable telehealth services has been presented. All the necessary features of the reviewed systems have been organized in Tables 1, 2, and 3 . 
Table 3 Available telehealth services based on Software

\begin{tabular}{|c|c|c|c|c|}
\hline Authors & Received data format & Transferring media & Major technology/sensor used & Provided services \\
\hline Magdalena et al. [42] & $\begin{array}{l}\text { Video conference } \\
\text { and electronic mail } \\
\text { with image data }\end{array}$ & NEMESIS database & Java programming language & ECG signal \\
\hline Mendes et al. [43] & Medical data & Cloud & $\begin{array}{l}\text { Wireless sensor network, VITABOX, BLE biomet- } \\
\text { ric sensors }\end{array}$ & $\begin{array}{l}\text { Blood pressure, } \\
\text { heart rate, weight, } \\
\text { oximetry or blood } \\
\text { glucose }\end{array}$ \\
\hline Jalil et al. [44] & Prenatal data & Cloud & N/A & Obstetrics patients \\
\hline Matsumoto et al. [45] & Any kinds of data & Cloud & Unified modeling language & Any kinds of patients \\
\hline Wang and Winters [46] & Medical data & XML & Embedded expert system, neurofuzzy technology & Any kinds of patients \\
\hline Doxy.me [47] & $\begin{array}{l}\text { Video, call, message, } \\
\text { screen, or file } \\
\text { sharing }\end{array}$ & Cloud & $\begin{array}{l}\text { Electronic health records and practice manage- } \\
\text { ment }\end{array}$ & Any kinds of patients \\
\hline AMC Health [48] & Any kinds of data & Cloud and bluetooth & Bluetooth & $\begin{array}{l}\text { Any kinds of } \\
\text { patients, especially } \\
\text { for COVID-19 } \\
\text { testing }\end{array}$ \\
\hline
\end{tabular}

N/A: not appropriately defined

The main features such as received data format, transferring media, major technology/ sensor used, provided services are focused on describing these systems.

As most of the reviewed scalable telehealth systems make the use of the already existing world-wide network infrastructure, patients around the world can get help from doctors and specialists regardless of their physical distance. The reviewed systems used various forms of data to exchange necessary information. Some systems collected data as an image format [27-29, 34, 37] and some systems in video [35, 37, 38], or voice call [26-29] from patient side. Some mobile network based telehealth systems also received SMS [24, 25] from patients. Whereas some frameworks [45-48] did not have any restrictions on the data format received from patient's devices.

The reviewed systems also used different types of networks to send and receive necessary information. Mobile network based systems used GSM network whereas some systems used GPRS [25], P2P [25], and 3G cellular network [27] as transferring medium. Social media based systems utilized their databases such as Facebook [36, 37], WhatsApp [34], email [35], etc. as data transferring medium on top of internet connectivity technologies. Similarly, software based systems used cloud and proprietary servers for data transferring purposes.

Almost all of the reviewed systems used various types of sensors to measure necessary data from the patient's body. The temperature sensor, glucose sensor, BP sensor, weight measure sensor, $\mathrm{SpO} 2$ sensor, and ECG machine have been generally used in the reviewed systems to monitor and test potential patients. While some reviewed systems are designed to provide general emergency support, some systems are proposed only for special cases such as obstetrics patients [44], chest problems [34], older adult patients' problems [37], and ECG monitoring [42]. Furthermore, the system reviewed in [48] provided a way to test potential COVID-19 patients from a safe distance.

As doctors and healthcare workers need to get close to potential patients infected with the COVID-19 virus for proper testing, they are more vulnerable to infection. Also, a lot of developing and under-developed countries do not have sufficiently advanced healthcare systems or expertise in a global pandemic. Telehealth technologies not only reduce the risk of infection of healthcare professionals, but also bridge the gap among countries to provide emergency services to people who need it regardless of nationality, gender, race, caste, or religion. The reviewed systems are scalable in the sense that, the systems can accommodate the growing number of infected patients by increasing the number of interested specialists and doctors in the platforms. However, most of the systems are depended on the doctor's or specialist's feedback. Modern machine learning [49] and artificial intelligence [50-52] technology may improve the quality of early detection and proper direction in healthcare and aid the doctors in faster detection. Proper guidelines to use telehealth technology alongside general clinical measures should also be developed to fight against the ongoing pandemic. Integrated telehealth systems including proper sensory and networking hardware can also be developed for providing proper support in rural areas.

Telehealth systems also have some limitations. If the patients themselves connect and measure the various information through the electronic sensors, the risk of improper setup or incorrect readings increases. Also, electronic 
devices and sensors have varying levels of measurement errors. As these telehealth systems exchange very sensitive medical information, data privacy is also a huge concern.

\section{Conclusion}

The number of COVID-19 infected patients is increasing exponentially around the world. To minimize the COVID transmission risk and maintaining social distance, scalable telehealth services play an important role to provide health services to the community. This paper overviews the available scalable telehealth services that are frequently used for COVID-19 patients as well as other patients. The existing telehealth systems are divided into mobile networks, social media, and software based services. The systems are described throughout the paper in terms of working principle, technology, used equipment, and types and methodology of service provided. The current challenges of existing technologies with possible future trends are also drawn in this review. Based on the findings of this study, clinicians and patients are highly recommended to apply telehealth systems as an appropriate option to prevent and avoid COVID-19 infection.

Funding No funding sources.

\section{Compliance with Ethical Standards}

Conflict of interest On behalf of all authors, the corresponding author states that there is no conflict of interest.

\section{References}

1. Monaghesh E, Alireza H. The role of telehealth during COVID-19 outbreak: a systematic review based on current evidence. BMC Public Health. 2020;20:1193.

2. Islam MM, Rahaman A, Islam MR. Development of smart healthcare monitoring system in IoT environment. SN Comput Sci. 2020;1(3): 185 .

3. Rahaman A, Islam M, Islam M, Sadi M, Nooruddin S. Developing IoT based smart health monitoring systems: a review. Rev d'Intelligence Artif. 2019;33(6):435-40.

4. Smith AC, et al. Telehealth for global emergencies: implications for coronavirus disease 2019 ( COVID-19). J Telemed Telecare. 2020a;26(5):309-13.

5. Perrone G, Zerbo S, Bilotta C, Argo A. Telemedicine during Covid-19 pandemic: advantage or critical issue ? Med Leg J. 2020;88(2):76-7.

6. Iyengar K, Mabrouk A, Kumar V, Venkatesan A. Learning opportunities from COVID-19 and future effects on health care system. Diabetes Metab Syndr Clin Res Rev. 2020;14(5):943-6.

7. Yellowlees P, Nakagawa K, Pakyurek M, Hanson A, Elder J, Kales HC. Rapid conversion of an outpatient psychiatric clinic to a $100 \%$ virtual telepsychiatry clinic in response to COVID-19. Psychiatr Serv. 2020;71(7):749-52.
8. Iyengar K, Upadhyaya GK, Vaishya R, Jain V. COVID-19 and applications of smartphone technology in the current pandemic. Diabetes Metab Syndr Clin Res Rev. 2020;14(5):733-7.

9. Zamberg I, Manzano S, Posfay-Barbe K, Windisch O, Agoritsas T, Schiffer E. A mobile health platform to disseminate validated institutional measurements during the COVID-19 outbreak: utilization-focused evaluation study. JMIR Public Heal Surveill. 2020;6(2):e18668.

10. Bassi A, Arfin S, John O, Jha V. An overview of mobile applications (apps) to support the coronavirus disease 2019 response in India. Indian J Med Res. 2020;151(5):468-73.

11. Pratap R, Javaid M, Kataria R, Tyagi M, Haleem A. Significant applications of virtual reality for COVID-19 pandemic. Diabetes Metab Syndr Clin Res Rev. 2020;14(4):661-4.

12. Wang SSY, Teo WZW, Teo WZY, Chai YW. Virtual reality as a bridge in palliative care during COVID-19. J Palliat Med. 2020;23(6):756-756.

13. Money P, April M, Leite H, Hodgkinson IR, Gruber T. New development: 'Healing at a distance '-telemedicine and COVID-19. Public Money Manag. 2020;40(6):483-5.

14. Vishal Dineshkumar Soni. Information technologies: shaping the world under the pandemic COVID 19. J Eng Sci. 2020;11(6):771-6.

15. Hong Y-R, Lawrence J, Williams D Jr, Mainous A III. Population-level interest and telehealth capacity of US hospitals in response to COVID-19: cross-sectional analysis of Google Search and National Hospital Survey Data. JMIR Public Heal Surveill. 2020;6(2):e18961.

16. Smith AC, et al. Telehealth for global emergencies: Implications for coronavirus disease 2019 (COVID-19). J Telemed Telecare. 2020b;26(5):309-13.

17. Neubeck L, Hansen T, Jaarsma T, Klompstra L, Gallagher R. Delivering healthcare remotely to cardiovascular patients during COVID-19. Eur J Cardiovasc Nurs. 2020;19(6):486-94.

18. Wosik J, et al. Telehealth transformation: COVID-19 and the rise of virtual care. J Am Med Inform Assoc. 2020;27(6):957-62.

19. Patel PD, et al. Rapid development of telehealth capabilities within pediatric patient portal infrastructure for COVID-19 care: barriers, solutions, results. J Am Med Inform Assoc. 2020;27(7):1116-20.

20. Oliver N, et al. Mobile phone data for informing public health actions across the COVID-19 pandemic life cycle. Sc Adv. 2020;6(23): eabc764.

21. Drew DA, et al. Rapid implementation of mobile technology for real-time epidemiology of COVID-19. Science (80-). 2020;368(6497):1362-7.

22. Trivedi A, Zakaria C, Balan R, Shenoy P. WiFiTrace: networkbased contact tracing for infectious diseases using passive WiFi sensing. May 2020. [Online]. arXiv:2005.12045.

23. Feng W, et al. Analysis of special ehealth service for corona virus disease 2019 (COVID-19) pneumonia. Beijing Da Xue Xue Bao. 2020;52(2):302-7.

24. García JE, Torres RA. Telehealth mobile system. In: 2013 Pan American health care exchanges (PAHCE). Medellin; 2013. pp. $1-5$.

25. Suganthi J, Umareddy NV, Awasthi N. Medical alert systems with TeleHealth \& telemedicine monitoring using GSM and GPS technology. In: 2012 3rd int. conf. comput. commun. netw. technol. ICCCNT 2012, no. July 2012.

26. Ade M, Doulamis N, Wagle SS, Ullah MG. TeleHealth: Healthcare technologies and TeleHealth Emergency (THE) system. In: 2011 2nd international conference on wireless communication, vehicular technology, information theory and aerospace \& electronic systems technology (Wireless VITAE), Chennai; 2011. pp. 1-4. 
27. Sorwar G, Ali A. Advanced telemedicine system using 3G cellular networks and agent technology. In: First IMIA/IFIP Jt. Symp. E-Health; 2014. pp. 187-97.

28. Mishra A, Agrawal DP. Continuous health condition monitoring by by $24 \times 7$ sensing and transmission of physiological data over 5-G cellular channels. In: 2015 International Conference on Computing, Networking and Communications (ICNC). IEEE: 2015. pp 584-590.

29. Kyriacou E, et al. Multi-purpose healthcare telemedicine systems with mobile communication link support. Biomed Eng Online. 2003;2:7.

30. Massaad E, Cherfan P. Social media data analytics on telehealth during the COVID-19 pandemic. Cureus. 2020;12(4):e7838.

31. Ni MY, et al. Mental Health, risk factors, and social media use during the COVID-19 epidemic and cordon sanitaire among the community and health professionals in Wuhan, China: CrossSectional Survey. JMIR Ment Heal. 2020;7(5):e19009.

32. Albarqouni L, Hoffmann T, McLean K, Price K, Glasziou P. Role of professional networks on social media in addressing clinical questions at general practice: a cross-sectional study of general practitioners in Australia and New Zealand. BMC Fam Pract. 2019;20:43.

33. Ohannessian R, Duong TA, Odone A. Global telemedicine implementation and integration within health systems to fight the COVID-19 pandemic: a call to action. JMIR Public Heal Surveill. 2020;6(2):e18810.

34. Davarpanah AH, et al. Novel screening and triage strategy in iran during deadly coronavirus disease 2019 (COVID-19) epidemic: value of humanitarian teleconsultation service. J Am Coll Radiol. 2020;17(6):734-8

35. Nicol GE, Piccirillo JF, Mulsant BH, Lenze EJ. Action at a distance: geriatric research during a pandemic. J Am Geriatr Soc. 2020;68(5):922-5.

36. Dhillon JS, Wünsche BC, Lutteroth C. Accessible telehealthLeveraging consumer-level technologies and social networking functionalities for senior care. In: 2013 6th international conference on human system interactions (HSI), Sopot; 2013. pp. 451-58.

37. Huang YC, Hsu YL. Social networking-based personal home telehealth system: a pilot study. J Clin Gerontol Geriatr. 2014;5(4):132-9.

38. Leung R, Guo H, Pan X. Social Media Users' perception of telemedicine and mHealth in China: Exploratory Study. JMIR mHealth uHealth. 2018;6(9):e181.

39. Vandekerckhove P, Vandekerckhove Y, Tavernier R, De Jaegher $\mathrm{K}$, de Mul M. Leveraging user experience to improve video consultations in a cardiology practice during the COVID-19 pandemic: initial insights. J Med Internet Res. 2020;22(6):e19771.
40. Li M, et al. Development of an openEHR template for COVID-19 based on clinical guidelines. J Med Internet Res. 2020;22(6):e20239.

41. Hoffman GJ, Webster NJ, Bynum JPW. A framework for agingfriendly services and supports in the age of COVID-19. J Aging Soc Policy. 2020;32(4-5):450-9.

42. Magdalena JR, et al. NEMESIS : a new telemedicine approach to cardiologic software. Comput Cardiol. 1999;26(Cat. No.99CH37004):37-40.

43. Mendes D, Jorge D, Pires G, António R, Dias P, Oliveira L. VITASENIOR-MT: a distributed and scalable cloud-based telehealth solution. In: 2019 IEEE 5th World Forum on Internet of Things (WF-IoT); 2019. pp. 767-72.

44. Jalil N, Member CP-I, Kadiman S, Member ES-I. Design and implementation of a telehealth platform for prenatal management in rural and remote areas. In: 2014 IEEE conference on biomedical engineering and sciences (IECBES); 2014. pp. 8-10.

45. Matsumoto T, Ogata S, Kawaji S. Designing and implementation of mobile terminal for telehealth care life support system. In: The 7th international conference on computer supported cooperative work in design, Rio de Janeiro, Brazil; 2002. pp 479-85.

46. Wang Y, Winters JM. Implementation of multimedia conferencing and neurofuzzy. In: Proceedings of the second joint 24th annual conference and the annual fall meeting of the biomedical engineering society engineering in medicine and biology]; 2002. pp. 1863-64.

47. Doxy.me. [Online]. https://doxy.me/. Accessed 24 Jul 2020.

48. AMC Health. [Online]. https://www.amchealth.com/covid-19/. Accessed 24 Jul 2020.

49. Muhammad LJ, Islam MM, Usman SS, Ayon SI. Predictive data mining models for novel coronavirus (COVID-19) infected patients' recovery. SN Comput Sci. 2020;1(4):206.

50. Islam MM, Karray F, Alhajj R, Zeng J. A review on deep learning techniques for the diagnosis of novel coronavirus (COVID-19). 2020. [Online]. arXiv:2008.04815.

51. Islam MZ, Islam MM, Asraf A. A combined deep CNN-LSTM network for the detection of novel coronavirus (COVID-19) using X-ray images. Inform Med Unlocked. 2020;20:100412.

52. Islam MM, Islam MZ, Asraf A, Ding W. Diagnosis of COVID-19 from X-rays using combined CNN-RNN architecture with transfer learning (2020). [Online]. https://www.medrxiv.org/conte $\mathrm{nt} / 10.1101 / 2020.08 .24 .20181339 \mathrm{v} 1$.

Publisher's Note Springer Nature remains neutral with regard to jurisdictional claims in published maps and institutional affiliations. 